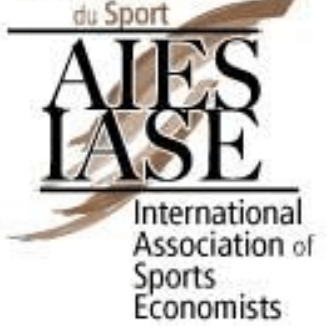

Working Paper Series, Paper No. 09-10

\title{
Anomalies in Tournament Design: The Madness of March Madness
}

\author{
Robert Baumann ${ }^{\dagger}$, Victor A. Matheson ${ }^{\dagger \dagger}$, and Cara Howe ${ }^{\dagger \dagger \dagger}$
}

October 2009

\begin{abstract}
Tournament design is of crucial importance in competitive sports. The primary goal of effective tournament design is to provide incentives for the participants to maximize their performance both during the tournament and in the time period leading up to the tournament. In spectator sports, a secondary goal of tournament design is to also promote interesting match ups that generate fan interest. Seeded tournaments, in general, promote both goals. Teams or individuals with strong performances leading up to a tournament receive higher seeds which increase their chances of progressing further in the tournament. Furthermore, seeding ensures that the strongest teams or players are most likely to meet in the final rounds of the tournament when fan interest is at its peak. Under some distributions of team or player skill, however, a seeding system can introduce anomalies that could affect incentives.

Our analysis of the NCAA men's basketball tournament uncovers such an anomaly. The seeding system in this tournament gives teams with better success in the regular season more favorable first round match ups, but the tournament is not reseeded as the games progress. Therefore, while higher seeds progress to the 2 nd round of the tournament at uniformly higher rates than lower seeds, this relationship breaks down in later rounds. We find that 10th and 11th seeds average more wins and typically progress farther in the tournament than 8th and 9th seeds. This finding violates the intended incentive structure of seeded tournaments.
\end{abstract}

JEL Classification Codes: L83, D02

Keywords: basketball, tournament design, sports, NCAA

'Robert Baumann, Department of Economics, Box 192A, College of the Holy Cross, Worcester, MA 01610-2395, 508-793-3879 (phone), 508-793-3708 (fax), rbaumann@holycross.edu

$\dagger$ Victor A. Matheson, Department of Economics, Box 157A, College of the Holy Cross, Worcester, MA 01610-2395, 508-793-2649 (phone), 508-793-3708 (fax), vmatheso@holycross.edu

${ }^{\dagger \dagger}$ Cara Howe, Department of Economics, College of the Holy Cross, Worcester, MA 01610-2395, cahowe10@holycross.edu 


\section{Introduction}

Tournament design is an integral part of the operation of any sports league or event, and contest design has been examined by numerous economists. Szymanski (2003) provides a comprehensive overview but notes that "issues deserving further attention include the value of screening [and] the role of handicapping.” This paper examines these issues by focusing on the structure of the National Collegiate Athletic Association's (NCAA) men’s basketball tournament, also known as “March Madness.”

A primary goal of effective tournament design is to provide incentives for the participants to maximize their performance both during the tournament and in the time period leading up to the tournament. In spectator sports, a second goal of tournament design is to promote interesting match ups that generate fan interest. Of course, a huge variety of contest designs can be utilized both in individual and team sports.

One broad type of tournament design is of the "round robin" tournament, or some variant, in which every participant plays games against each of the other contestants. Most regular season games in sports leagues utilize a variation of the round robin tournament. In a round robin tournament, teams or individuals play a number of matches against different opponents with the winner of the most games being declared the victor. For example, in the overwhelming majority of European soccer leagues each team plays one game at home and one game on the road against each of the other teams in the league. In a 20-team league like the English Premier League or Spain’s La Liga, this results in a 38 game schedule for each team with the most successful team being named the annual league champion. 
The majority of American professional sports leagues utilize unbalanced round robin tournaments for their regular season contests where teams play many or all of the other teams in the league but play more games against geographical or historical rivals. In the National Football League, for example, each team plays a home and away series with each team in its own division and then games against 10 of the remaining 28 teams in the league. In the National Basketball Association, each team plays two home and away series with every team in their own division, either 3 or 4 games against every remaining team in their conference, and a single home and away series with each team in the opposite conference. In most American professional sports leagues, the top teams then qualify for a post-season playoff tournament

The other major category of tournament is the "elimination tournament” where contestants match up against one another and the winners progress while the losers are eliminated from further participation in the tournament. As with round robin tournaments, numerous variations on this the concept exist. In a standard single elimination tournament, a single game determines which player or team will advance. Both the NFL's post-season playoff system as well as the National Collegiate Athletic Association's (NCCA) men’s and women's basketball tournaments are set up as single elimination tournaments. Most tennis tournaments and match-play golf tournaments are also examples of simple single elimination tournaments. Another common variant on the elimination tournament uses series of games between two opponents to determine who advances in the tournament. For example, in the NBA the winner of a best of seven series goes through to the next round, while in some stages of the European Champions League in soccer as well as many levels of the World Cup qualifying tournaments, the winner of 
a home and away series advances. A third variant is the "double elimination” tournament where a team is removed from the tournament once it has lost two contests.

Finally, many sports utilize a combination of round robin and single elimination tournament. For example, as noted previously, American sports leagues typically use a round robin format during the regular season and then progress to an elimination tournament for playoffs. The FIFA World Cup uses four-team round robin tournaments in the first round of the competition before progressing to a 16-team single game elimination format.

\section{Screening, Seeding, and Handicapping}

Regardless of the type of tournament design used, a variety of methods can be used to determine which teams or players will play one another. Screening refers to which teams or players will be eligible for a competition. Seeding and handicapping refers to placement of teams that have been selected for a tournament. Of course, tournaments themselves are frequently used for screening purposes for future tournaments. Regular season results are used as screening for playoffs in American leagues or for advancement into European-wide tournaments such as the UEFA or Champions League tournaments in European soccer. Confederation results are used for screening advancement into the World Cup. In leagues with promotion and relegation, a prior season's record serves as a screening device for which league a team will play in during the current season. Similarly, in the Professional Golfers Association (PGA), players with a certain level of winnings during the previous season are eligible to compete in professional tournaments during the current season. 
Once screening has been achieved, in a full round robin tournament, all teams play an identical schedule, so seeding or handicapping the teams in the tournament is unnecessary. In other tournament designs, however, decisions must be made about how match ups will be set. In some cases, competition choices will be made without specific consideration of the skill of the teams or players involved. For example, in leagues such as the NBA and MLB, where an unbalanced schedule is played, the implementation of the schedule does not generally depend on the prior success of the teams involved but instead depends largely on geography and historical accident. Similarly, some elimination tournaments use a random draw where match ups are done by chance without consideration of the skill of the teams or players involved. The initial groups for round robin play in the World Cup are chosen at least in part by chance during the highly anticipated World Cup draw. While teams are placed in various equivalence classes based on performance, within each class, teams are chosen at random. Given the existence of variation in skills within each class, pure luck can result in some four-team groups ending up with stronger teams on average than other groups. Every country dreads being randomly placed in the so-called “Group of Death” with the strongest teams on average.

Conversely, tournaments can be designed with team or player strength in mind. In the NFL, the league uses a weighted schedule to promote competitive balance. All teams play a full round robin schedule with the other 3 teams in their division, and divisions are created based upon geography and historical rivalry, but the remaining 10 games of the season are scheduled based upon the previous season's standings with stronger teams 
being scheduled against better opponents. In the case of the NFL, strong teams are "rewarded" for their success by playing a harder schedule in the following season.

More commonly, good teams or players are rewarded for their success through seeding. In both round robin and elimination tournaments, prior success can be accounted for by the use of seeding, where top seeded players are assigned to play lower quality teams or players in early rounds. Playoffs in American professional leagues are typically fully seeded tournaments where the team with the best record in the regular season plays in the first round the team with the worst record in the regular season that qualified for the playoffs. The NCAA men's and women's basketball tournaments are also fully seeded in this fashion.

Other tournaments may be partially seeded with only a portion of the field receiving preferential treatment in this fashion. As noted previously, the FIFA World Cup is partially seeded with teams being placed within categories by skill but with teams not being ranked within each category. In the NCAA men's and women's soccer tournaments, only the top 16 teams in each gender are seeded with other match ups being made with travel considerations in mind.

Alternatively, seeded players or teams can receive "byes" into later rounds of the tournament. A bye means a competitor is exempt from playing a game or games in the early rounds of a tournament and effectively works as an automatic advancement out of the early stages of the contest. In the NFL, the top two division champions in each conference each receive a bye into the second round of the playoffs, while the bottom two division champions play the against the top two non-division champions, known as wildcard teams, from each conference in the first round of the playoffs. Thus, a wildcard 
team or bottom two division champion team need to win 4 games in the playoff to be declared champion, while each of the top two division leaders in each conference needs only to win three games to take home the crown due to the bye.

In the England Football Association (FA) Cup, a competition that pits teams at all levels of skill against one another, teams from the country’s highest professional division, the English Premier League receive multiple rounds of byes before entering into the tournament making the path to the trophy for a semi-professional underdog team far longer than that of a team like Manchester United or Chelsea.

\section{Seeding vs. Re-Seeding}

Tournament seedings generally promote two important goals of sports organizers. First, seeded tournaments promote effort during the time period prior to the tournament since teams or individuals with strong performances leading up to a tournament receive higher seeds which increase their chances of progressing further in the tournament. If league playoffs were randomly seeded, for example, teams would have little incentive to win games late in the season once they had clinched a playoff berth. Furthermore, seeding ensures that the strongest teams or players are most likely to meet in the final rounds of the tournament during which time fan interest is at its peak.

Most post-season tournaments have multiple rounds, and many seeding plans reward teams or individuals with more favorable match ups not only in the first round but also in later rounds. For example, in the NFL playoffs, the top overall seed in each conference receives a bye in the first round of the tournament and then earns the right to play the lowest remaining seed after the first round of games are played. Not all 
tournaments update their seeds, or "re-seed”, each round, however. Without re-seeding at each round, the higher ranked teams are not guaranteed to play the weakest remaining teams because of upsets. The World Cup, NBA playoffs, NCAA basketball tournament, and most professional tennis events are examples of leagues without re-seeding. In some cases, this lack of re-seeding lowers travel costs. For example, the NBA produces separate seedings for teams in the Eastern and Western conferences, and the winner of each conference faces each other in the finals. This also ensures fan interest in the finals will not be limited one geographic area. But cases like the World Cup and NCAA basketball tournament, no such cost advantages exist. Other leagues such as the NHL and NFL re-seed at each round, guaranteeing the highest ranked team will play the lowestranked remaining opponent. While this produces more uncertainty about future opponents for players and fans, it could affect incentives for teams prior to the postseason. We consider the example of NCAA Men’s Basketball that produces this anomaly in tournament design.

\section{NCAA Men’s Basketball}

There are 347 NCAA Division I basketball programs. Each team plays roughly 30 games during the regular season. There are two ways teams can qualify for the postseason tournament also known as “March Madness”. With the exception of the relatively new Great Western Conference, teams that win their conference and given an automatic bid in the tournament. All but one of the 31 conferences determines their automatic bid with a conference tournament at the end of the regular season. In the remaining conference, the Ivy League, the team with the best regular season conference record earns 
the automatic bid. The remaining 34 March Madness spots are called "at-large” bids, which are determined by the NCAA Selection Committee. The ten-member committee is usually comprised of athletic directors and conference administrators. This committee selects the best 34 non-conference winners.

Once the 65 teams are chosen, four "regions" are created with 16 teams. The two teams determined to be the worst of the 65 compete in a "play-in" game before the rest of the field. The winner of the play-in game then advances to the field of 64, leaving four equal regions of 16 teams. Regions are defined by the location of the games, which are held on neutral courts. Typically only the highest ranked teams are given geographic preference to play closest to home, and remaining teams are distributed randomly across the country. Teams within each region are ranked 1 through 16, giving the highest ranked team the privilege of playing the lowest ranked opponent in their region. This pattern continues throughout the rankings, e.g. the second seed plays number 15, the third seed plays number 14, and so on. The bracket is also set up to benefit higher ranked teams in later rounds. Assuming a win in the initial round, the number one seed will play either the 8 or 9 seed, the number two seed will play either the 7 or 10 seed, and so on. The tournament is single-elimination, resulting in six rounds not counting the play-in game.

March Madness does not re-seed with each round, and upsets may produce lessfavorable match ups for higher ranked teams in later rounds. For example, if the favored team wins in each round, in the second round the number 1 seed will play the number 8 seed and the number 2 seed will play the number 7 seed. In the event of an upset of the number 2 seed in the first round, however, the number 1 seed would still play the number 8 seed, and the number 7 seed would play the number 15 seed. Under specific 
distributions of talent across teams, one could end up with the situation that fair seedings in the first round actually lead to lower seeds advancing further in the tournament than high seeds.

Table 1 and Figure 1 show the distribution of wins in the first round by seed for all NCAA men’s tournaments since March Madness expanded to 64 teams in 1986. In total, the data set encompasses 96 matches at each seed (24 years and 4 games for each seeding pair per year). The data show that the tournament committee performs well in their initial seedings as the win percentages generally fall uniformly over the seeds. The exceptions are that number 5 seeds have performed below expectations and number 9 seeds have a small, but statistically insignificant advantage over number 8 seeds. Overall, however, as a team's initial seed rises, the team has a smaller chance of advancing to the second round, as one would expect from a properly seeded tournament.

Since the tournament is not reseeded at each round, however, advancement out of the second round is a far different story. While a number 10,11, 12 seed has a lower chance of advancing to the second round than an 8 or 9 seed, their chances of advancing to the third round are much higher than those of 8 or 9 seeds. In fact, number 10 seeds have advanced to the third round, known as the sweet sixteen, 6 times as often as number 9 seeds and over twice as often as number 8 seeds. This surprising result is easily explained by the lack of reseeding. First, while number 10 seeds are less likely to advance to the $2^{\text {nd }}$ round than a number 8 or 9 seed, once they get there they will face a number 2 seed or perhaps even a number 15 seed in the event of a first-round upset. An 8 or 9 seed will almost certainly face a tougher $2^{\text {nd }}$ round opponent since number 1 seeds are stronger than number 2 seeds and number 1 seeds are less likely to be upset in the 
first round. Similarly, number 11 and 12 seeds likely face weaker number 3 or number 4 seeds, respectively, in the second round and are far more likely to benefit from first round upsets than number 8 and 9 seeds. These advantages in the second round outweigh the disadvantages seeds 10 through 12 face in the first round of the competition.

\section{Conclusions and Policy Suggestions}

Colleges and universities have several reasons for promoting intercollegiate athletics. A successful program can generate revenues for the school or the conference either directly through sports revenue or indirectly through alumni donations. In term of direct revenue, the NCAA redistributes half of the profits from the tournament directly to conferences based on the number of games conference members appear in during the tournament. Each appearance by a conference member over the previous 6 seasons entitles the conference to one share of this profit pool. Considering that the tournament generates profits in excess of \$300 million annually, in 2008-09 a single share was worth $\$ 206,000$. Since the NCAA uses a 6-year rolling window for profit distribution, this means that a single win by a team guarantees at least an extra $\$ 1.2$ million to the school's conference over the ensuing 6 years. On this criterion alone, the extra wins expected by a number 10 seed compared to a number 8 or 9 seed results in an expected $\$ 111$ thousand bonus to the conference if one of its teams is seeded 10 instead of 8 or 9 .

In addition, a team that goes deep into the NCAA tournament generates a large amount of publicity for the school and potentially increases both the number and the quality of applicants to the school as well as inducing higher alumni giving. For example, North Carolina State University experienced a 40\% increase in applications after their 
surprise victory in the 1983 NCAA Men’s Basketball Championship game. (McEvoy, 2005) Systematic proof of basketball's effect on admissions or alumni giving has proven more elusive, however. While numerous studies have demonstrated positive effects of football success on both giving and applications (e.g. McCormick and Tinsley (1987), Tucker and Amato (1993), Siegelman and Brookheimer (1983), Rhodes and Gerking (2000), and Baade and Sundberg (1994)), these studies have generally failed to uncover any impact of basketball success on these variables. Still, if such effects do exist, given the nature of media coverage, an appearance in the sweet sixteen is significantly more valuable than an appearance in the $2^{\text {nd }}$ round.

By both measures, the data highlight the obvious anomaly in the men's NCAA tournament that it is much better to be a number 10 seed than a number 8 or 9 seed and potentially better to be a 10,11 or 12 than an 8 or 9 . Number 10 seeds average more wins per tournament than 8 or 9 seeds ( 0.68 to 0.61 and 0.58 , respectively) and are more likely to reach the sweet sixteen (18.75\% to $8.33 \%$ and 3.13\%, respectively). Number 8 and 9 seeds win slightly more games per tournament than number 11 and 12 seeds, thus guaranteeing a higher NCAA payout for their conference, but 8 and 9 seeds are significantly lees likely to advance to the sweet sixteen than 11 or 12 seeds. Thus, the NCAA Men's Basketball Tournament appears to violate a fundamental property of tournament design that better seeded teams received higher expected rewards than lower seeded one.

The policy prescriptions for the NCAA are not obvious, however. It is perhaps reasonable for the NCAA to consider reseeding teams at each round, but this brings up two difficulties. First, such a policy would create travel difficulties. Only 48 hours 
separates the first two rounds of games. Reseeding would cause teams and spectators to have to make last minute travel plans both increasing cost and potentially reducing demand. This is particularly difficult in the March Madness tournament because all games are played at neutral sites. In the NFL playoffs, fans have a full week between games, so reseeding poses less difficulties. In the NBA playoffs, teams play a home and away series so fewer fans are traveling to away games, again reducing any difficulties caused by reseeding.

Of potentially greater concern, although the NCAA would be loathe to admit this, is the fact that reseeding would play havoc with the gambling markets. March Madness represents one of the largest venues for sports betting, both legal and illegal, in the country. The most common type of betting on the tournament consists of filling out full tournament brackets. Of course, such an activity requires that brackets remain fixed for the duration of the tournament. A reseeded tournament would eliminate this popular gambling option potentially reducing fan interest in the tournament. It is therefore likely that the NCAA Men’s Basketball Tournament will remain a statistical oddity. 


\section{References}

Baade, R. and Sundberg, J. (1994). Fourth down and gold to go? Assessing the link between athletics and alumni giving. Social Science Quarterly 77(4), 789-803.

McCormick, R. E. and Tinsley, M. (1987). Athletics versus academics? Evidence from SAT cores. Journal of Political Economy 95, 1103-1116.

McEvoy, Chad. 2005. The Relationship Between Dramatic Changes in Team Performance and Undergraduate Admissions Applications, The SMART Journal. Vol. 2:1, pp. 17-24.

Rhoads, T. and Gerking, S. (2000) Educational contributions, academic quality, and athletic success. Contemporary Economic Policy 18(2), 248-258.

Siegelman, L. and Bookheimer, S. (1983). Is it whether you win or lose? Monetary contributions to big-time college athletic programs. Social Science Quarterly 64(2), 347-359.

Tucker, I. B. and Amato, L. (1993). Does big-time success in football or basketball affect SAT scores? Economics of Education Review 12 (2), 177-181.

Szymanski, Stefan. 2003. The Economic Design of Sporting Contests, Journal of Economic Literature, Vol. 41:4, pp. 1137-1187. 
Table 1: Chance of advancing to future rounds by seed

Round

$\begin{array}{rrrrrrrc}\text { Seed } & & \mathbf{1} & \mathbf{2} & \mathbf{3} & \mathbf{4} & \mathbf{5} & \begin{array}{c}\text { Avg. wins } \\ \text { per seed }\end{array} \\ \mathbf{1} & 100.00 \% & 88.54 \% & 70.83 \% & 44.79 \% & 23.96 \% & 15.63 \% & 3.44 \\ \mathbf{2} & 95.83 \% & 63.54 \% & 45.83 \% & 21.88 \% & 11.46 \% & 4.17 \% & 2.43 \\ \mathbf{3} & 84.38 \% & 52.08 \% & 26.04 \% & 12.50 \% & 8.33 \% & 3.13 \% & 1.86 \\ \mathbf{4} & 79.17 \% & 43.75 \% & 14.58 \% & 9.38 \% & 2.08 \% & 1.04 \% & 1.50 \\ \mathbf{5} & 65.63 \% & 35.42 \% & 5.21 \% & 4.17 \% & 2.08 \% & 0.00 \% & 1.13 \\ \mathbf{6} & 70.83 \% & 36.46 \% & 12.50 \% & 3.13 \% & 2.08 \% & 1.04 \% & 1.26 \\ \mathbf{7} & 59.38 \% & 17.71 \% & 6.25 \% & 0.00 \% & 0.00 \% & 0.00 \% & 0.83 \\ \mathbf{8} & 46.88 \% & 8.33 \% & 4.17 \% & 2.08 \% & 0.00 \% & 0.00 \% & 0.61 \\ \mathbf{9} & 53.13 \% & 3.13 \% & 1.04 \% & 0.00 \% & 0.00 \% & 0.00 \% & 0.57 \\ \mathbf{1 0} & 40.63 \% & 18.75 \% & 8.33 \% & 0.00 \% & 0.00 \% & 0.00 \% & 0.68 \\ \mathbf{1 1} & 29.17 \% & 9.38 \% & 4.17 \% & 2.08 \% & 0.00 \% & 0.00 \% & 0.44 \\ \mathbf{1 2} & 34.38 \% & 16.67 \% & 1.04 \% & 0.00 \% & 0.00 \% & 0.00 \% & 0.52 \\ \mathbf{1 3} & 20.83 \% & 4.17 \% & 0.00 \% & 0.00 \% & 0.00 \% & 0.00 \% & 0.25 \\ \mathbf{1 4} & 15.63 \% & 2.08 \% & 0.00 \% & 0.00 \% & 0.00 \% & 0.00 \% & 0.18 \\ \mathbf{1 5} & 4.17 \% & 0.00 \% & 0.00 \% & 0.00 \% & 0.00 \% & 0.00 \% & 0.04 \\ \mathbf{1 6} & 0.00 \% & 0.00 \% & 0.00 \% & 0.00 \% & 0.00 \% & 0.00 \% & 0.00\end{array}$


Figure 1: Probability of Advancement to $2^{\text {nd }}$ round

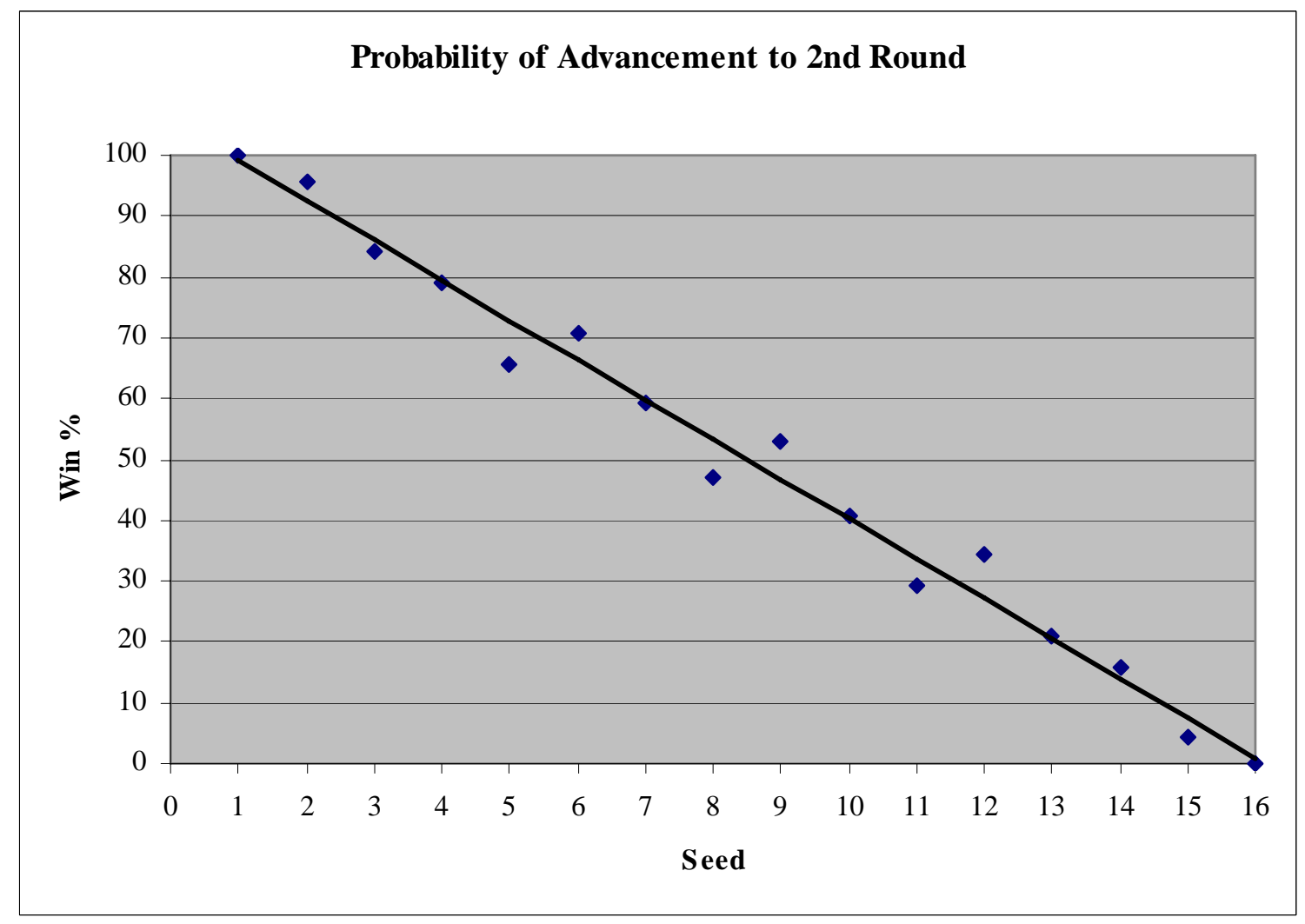

Figure 2: Probability of Advancement to $3^{\text {rd }}$ round

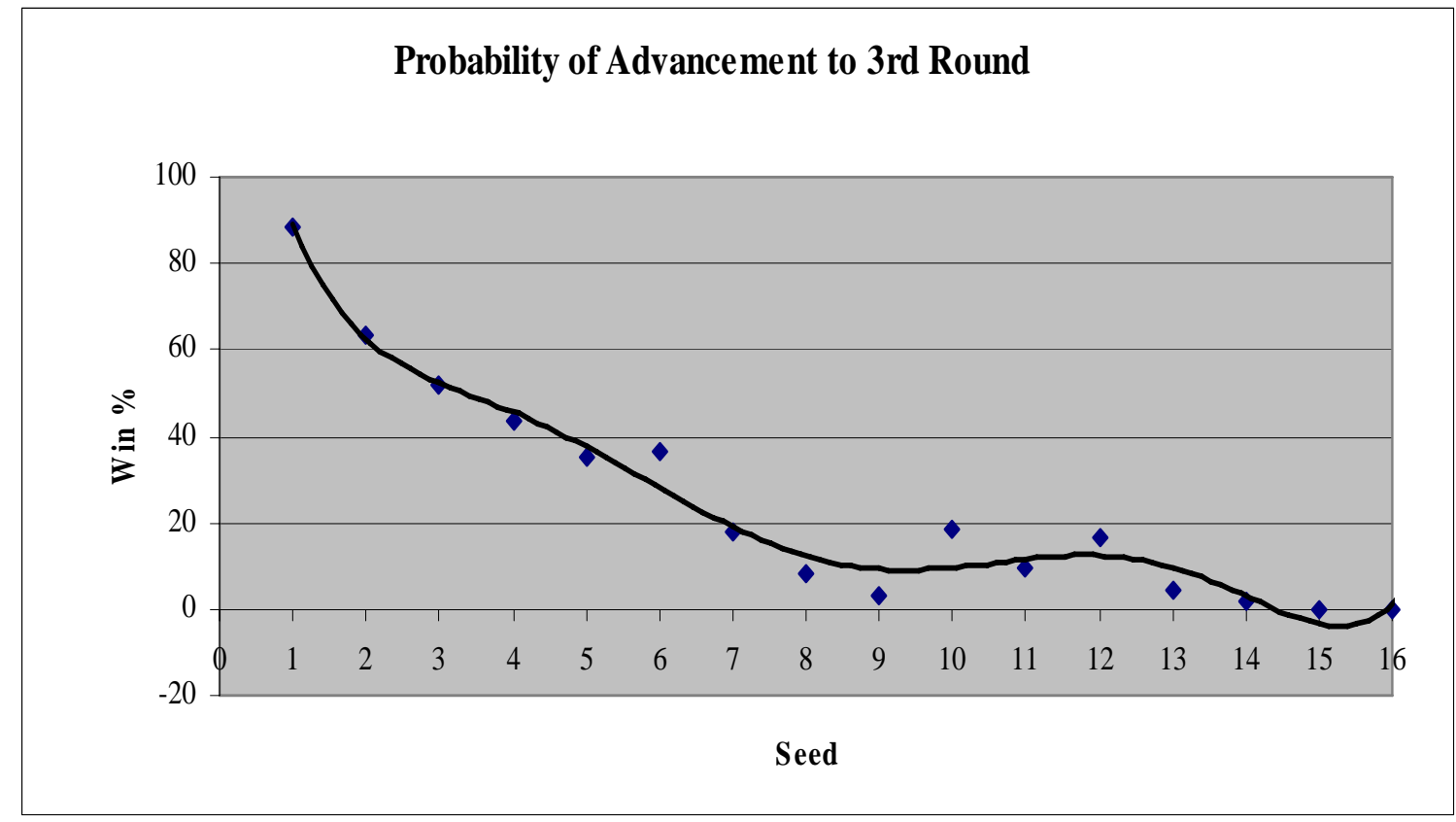

